\title{
Relação entre o aleitamento materno exclusivo nos seis primeiros meses e a prevenção da obesidade infantil
}

\author{
Relationship between exclusive breastfeeding in the first six months and the prevention of \\ childhood obesity
}

\section{Relación entre la lactancia exclusiva en los primeros seis meses y la prevención de la obesidad infantil}

Danyella Oliveira de Paula ${ }^{1 *}$, Carolina Alves Quintanilha², Carolina Felix de Sousa Chaer ${ }^{3}$, Hanna Barreto Dias ${ }^{4}$, Heloísa Fantucci Pascoal Vieira ${ }^{5}$, Jéssica Carvalho Buzzo ${ }^{6}$, Letícia Fernandes Braga da Mata ${ }^{7}$, Mariana Superbi Ferreira Barros ${ }^{8}$, Maryana Duarte Costa ${ }^{2}$, Rhamai Carneiro de Souza ${ }^{9}$.

\section{RESUMO}

Objetivo: Analisar e descrever a relação entre a amamentação e a prevenção da obesidade infantil para incentivar e estimular a amamentação, reduzindo assim também a incidência de doenças crônicas na fase adulta. Revisão bibliográfica: O aleitamento materno exclusivo para crianças até os 6 meses de vida é extremamente importante, visto que essa alimentação pode prevenir a obesidade infantil e outras comorbidades. Os "primeiros 1000 dias" entre a gravidez e os 24 meses de vida são eficazes para o desenvolvimento alimentar, visto que os nutrientes como aminoácidos e vitaminas que a criança recebe durante a gestação são necessários ao longo da sua vida. O leite materno possui imunoglobulinas que são relevantes na prevenção de infecções da primeira infância. Estudos mostram que quando há introdução de alimentos sólidos precocemente tem-se aumento no peso corporal e na estatura da criança podendo trazer uma consequência na vida adulta. Considerações finais: O leite materno é uma fonte fundamental e rica em nutrientes para os lactantes, importante tanto para o seu desenvolvimento quanto para a memória imunológica e prevenção de doenças crônicas.

Palavras-chave: Aleitamento materno, Obesidade pediátrica, Prevenção de doenças.

\begin{abstract}
Objective: Analyze and describe the connection between breastfeeding and the prevention of obesity to encourage and stimulate even more breastfeeding, reducing the incidence of chronic disease. Bibliographic review: The breastfeeding exclusive for children newborn until 6 months old is extremely important. This unique diet can prevent childhood obesity and other comorbidities. The "firsts 1000 days" between the pregnancy and the 24 months of life are very effective in food development. The nutrients like amino acids and vitamins that the child gets during gestation are so necessary throughout life. Breast milk has the presence of immunoglobulins that is relevant in the prevention of infections in early childhood. The weaning of the child must be a natural way and not force being able to bring loss for the mother and the child. Studies show that when exists premature nutrition with solid food there is a growth in the child's body weight and stature bringing consequences in adulthood. Final considerations: Breast milk is a fundamental source and very abundant of nutrients for lactating it's important both for their development and immune memory and prevention of chronic diseases.
\end{abstract}

Keywords: Breast feeding, Pediatric obesity, Disease prevention.

\footnotetext{
${ }^{1}$ Centro Universitário do Planalto Central Apparecido dos Santos (UNICEPLAC), Brasília - DF.

*E-mail: dany-lella@hotmail.com

2 Centro Universitário Presidente Antônio Carlos (UNIPAC), Juiz de Fora - MG.

3 Centro Universitário de Brasília (UniCEUB), Brasília - DF.

4 Universidade de Rio Verde (UNIRV), Formosa - GO.

${ }^{5}$ Centro Universitário Integrado (CEI), Campo Mourão - PR.

${ }^{6}$ Centro Universitário Católico Unisalesiano Auxilium (UNISALESIANO), Araçatuba - SP.

7 Faculdade Ciências Médicas de Minas Gerais (FCMMG), Belo Horizonte - MG.

8 Faculdade de Minas (FAMINAS), Belo Horizonte - MG.

${ }^{9}$ Faculdade de Medicina Estácio Juazeiro do Norte (Estácio FMJ), Juazeiro do Norte - CE.
} 


\section{RESUMEN}

Objetivo: Analizar y describir la relación entre la lactancia materna y la prevención de la obesidad para fomentar y estimular, aún más, la lactancia materna, reduciendo así, la incidencia de enfermedades crónicas. Revisión bibliográfica: La lactancia materna exclusivamente para niños recién nacidos hasta los 6 meses de edad es extremadamente importante. Esta dieta única puede prevenir la obesidad infantil y otras comorbilidades. Los "primeros 1000 días" entre el embarazo y los 24 meses de vida, son muy efectivos para el desarrollo de la alimentación. Los como aminoácidos y vitaminas que recibe el niño durante el embarazo son muy necesarios durante toda su vida. La leche materna tiene la presencia de inmunoglobulinas, lo que es relevante para prevenir las infecciones de la primera infancia. El destete del niño debe ser natural y no forzado, lo que puede dañar tanto a la madre como al niño. Los estudios demuestran que cuando hay una nutrición temprana con alimentos sólidos, hay un aumento en el peso corporal y la estatura del niño, lo que puede tener consecuencias en la edad adulta. Consideraciones finales: La leche materna es una fuente fundamental y muy rica de nutrientes para las mujeres lactantes. Es importante tanto para su desarrollo como para la memoria inmunológica y prevención de enfermedades crónicas.

Palabras clave: Lactancia materna, Obesidad pediátrica, Prevención de enfermedades.

\section{INTRODUÇÃO}

No último século, a obesidade infantil tornou-se problema de saúde mundial. Estima-se que 42 milhões de crianças abaixo dos 5 anos de idade estão acima do peso da média global. $O$ aumento da incidência dessa patologia em crianças gerou necessidade de intervenção nutricional durante esse período e, apesar da condição multifatorial da obesidade, sabe-se que essa possui forte relação com os aspectos nutricionais e metabólicos (SYMON B, et al., 2017).

Contribui também para o cenário de agravo à saúde o fato de já ser estudado que as consequências da obesidade infantil podem persistir até a vida adulta, pois o excesso de peso relaciona-se com 0 desenvolvimento precoce de patologias crônicas, como diabetes, hipertensão, dislipidemias e doenças cardiovasculares (MAMELI C, et al., 2016).

O estado nutricional na população pediátrica tem como "marcador" as curvas de crescimento preconizadas pela Organização Mundial de Saúde (OMS), de modo que a ingestão inadequada de nutrientes básicos pode influenciá-las, sendo o sobrepeso em crianças definido como um índice de massa corporal (IMC) no percentil 85 e a obesidade definida como um IMC no percentil 95 (podendo esses valores de referência serem superiores de acordo com a idade e sexo) (CORKINS MR, et al., 2016). Deve-se ressaltar que a primeira infância é um estágio em que o cuidado alimentício é de extrema necessidade, visto que nessa etapa, o excesso de peso pode ser evitado, não causando injúrias futuras (ARDID C, et al., 2019).

A nutrição infantil adequada, em especial nos primeiros anos de vida, influencia diretamente na prevenção de doenças crônicas durante essa fase e também na fase adulta (WEBER M, et al., 2014). A amamentação é um dos fatores mais amplamente discutidos e reconhecidos pelo seu papel protetor no histórico nutricional da obesidade na faixa etária pediátrica (MANNAN H, 2018). Assim, o leite materno é considerado padrão ouro na alimentação infantil, sendo recomendado pela OMS o aleitamento materno exclusivo até os 6 meses de vida e sua continuidade parcialmente por até 2 anos ou mais, juntamente com a introdução dos alimentos sólidos (SHAMIR R, 2016).

A amamentação é um conjunto de interações nutricionais, ambientais, socioeconômicas, psicológicas e genéticas que beneficia tanto a saúde do bebê, quanto da mãe. Evidências na literatura demonstram benefícios relacionados à imunidade do lactente, trazendo uma proteção contra infecções respiratórias superiores, pneumonia, otite média, infecções do trato urinário, sepse e meningite. Além disso, reduz o risco de desenvolver patologias como a obesidade, asma, alergia, eczema, dermatite atópica, doenças gastrointestinais, diabetes mellitus, hipertensão e até mesmo a incidência de morte súbita na infância (SHAMIR R, 2016).

Entretanto, novas rotinas, hábitos e exigências culturais do mundo moderno influenciam as escolhas das mães em relação à introdução precoce de fórmulas e sólidos, o que gera um risco maior de diagnóstico de sobrepeso e obesidade infantil, podendo essa associação ser modificada pela amamentação e pelo tempo de aleitamento exclusivo (HARRISON M, et al., 2016; MANNAN H, 2018). 
Algumas hipóteses foram levantadas e analisadas no que tange o efeito protetor do aleitamento materno em relação ao excesso de peso como, por exemplo, comparação das curvas de crescimento; o conteúdo do leite materno versus a fórmula, analisando especialmente a ingestão precoce de proteínas e a variabilidade do conteúdo que influencia a seletividade de alimentos saudáveis na infância; respostas metabólicas e endócrinas diferentes; a introdução alimentar precoce de sólidos; a duração da amamentação e sua exclusividade, entre outros (CORKINS MR, et al., 2016; HASCHKE F, et al., 2016; SPECHT IO, et al., 2018; SYMON B, et al., 2017; VALDÉS JMB, et al., 2018).

A realização desse estudo justifica-se com o intuito de contribuir para o incentivo da amamentação, dada a sua importância baseada em evidências científicas, visto que esta proporciona às crianças muitos benefícios e proteção contra várias doenças, porém, enfatizando a obesidade neste estudo, uma vez que existe uma grande incidência e prevalência de tal doença na população pediátrica que, consequentemente, se estende à vida adulta. Diante do exposto, o objetivo da presente revisão de literatura é analisar e descrever a associação entre o aleitamento materno e a prevenção da obesidade, a fim de fomentar e incentivar ainda mais a amamentação e consequentemente diminuir a incidência desta doença crônica tão prevalente.

\section{REVISÃO BIBLIOGRÁFICA}

A obesidade é uma doença que vem se expandindo em proporção mundial, inclusive entre as crianças. Por isso, tal patologia é considerada pela Organização Mundial da Saúde (OMS) uma epidemia mundial desde os anos 90 (LOPES AF, et al., 2016). Mesmo que na atualidade tenha-se maior consciência social e significativas intervenções preventivas de saúde pública, a obesidade infantil permanece sendo um assunto de importância global (MAMELI C, et al., 2016).

Em relação aos dados epidemiológicos, tem-se que nos Estados Unidos há uma prevalência, dentro da faixa de sobrepeso para obesidade, maior que 30\% em crianças que não tiveram aleitamento materno exclusivo no primeiro semestre de vida. Apesar dos avanços e conhecimentos sobre o tema, bem como maior conscientização da população a respeito da amamentação e obesidade, essa prevalência apresentou índice crescente nos últimos vinte anos e segue uma previsão de crescimento nas próximas décadas. Além disso, países de baixo e médio nível de desenvolvimento também relatam aumento na taxa de obesidade infantil, entretanto os mesmos países ainda têm um maior índice de desnutrição que supera a obesidade, mas também alertam para uma epidemia dessa patologia crônica infantil, relacionada com a alimentação, sobretudo nos primeiros anos de vida (MAMELI C, et al., 2016; HORN LV, et al., 2018).

A obesidade apresenta grande impacto na vida do indivíduo, pois é fator de risco para alterações metabólicas, doenças cardiovasculares e psicológicas, com consequências potencialmente devastadoras, inclusive na vida adulta (MAMELI C, et al., 2016). Ademais, essa patologia na primeira infância é sinal de alerta devido ao fato de que a grande parte das crianças obesas tendem a permanecerem com excesso de peso ou obesidade até a vida adulta e, consequentemente, têm o risco aumentado de doenças cardiovasculares e diabetes mellitus (SYMON B, et al., 2017).

Devido ao fato da obesidade atingir várias faixas etárias, inclusive a pediátrica, muitas pesquisas têm como objetivo traçar quais são os fatores de riscos que estão possivelmente associados a essa patologia. Dentre os fatores de risco listados para o precoce desenvolvimento da obesidade, têm-se o sexo, o peso ao nascer, a duração do aleitamento materno e o período de introdução alimentar complementar (LOPES AF, et al., 2016).

Alguns dados descritos na literatura, inclusive de estudos brasileiros, apontam que crianças com maior peso ao nascimento têm maior risco para o desenvolvimento de excesso de peso. Por outro lado, o sexo masculino é visto como fator de proteção. Em relação, a duração do aleitamento materno, quando a mesma é mais prolongada, se mostra como um fator de proteção para o desdobramento da obesidade. Complementar a isso, estudos americanos observaram que a menor duração do aleitamento materno equivale a um risco elevado do bebê apresentar excesso de peso (LOPES AF, et al., 2016).

Nos últimos 10 anos, as bactérias, sobretudo do trato gastrointestinal, ganharam o reconhecimento de cientistas em relação ao metabolismo e proteção do hospedeiro. A partir disso, o microbioma maduro do intestino humano, atualmente é considerado como um órgão auxiliar do hospedeiro e que apresenta íntima 
relação com a saúde e o bem-estar do indivíduo. E por isso, a composição da microbiota intestinal é também um fator preditor na evolução do peso do bebê, contudo, essa depende diretamente da dieta, acometimentos infecciosos, estilo de vida e hábitos cotidianos que fazem parte da vivência do bebê e seus familiares. Em países desenvolvidos, as doenças infecciosas foram reduzidas e controladas por uso de políticas públicas, que contribuíram positivamente para a redução de infecções e melhora da qualidade de vida, apesar de ainda não ter total adesão do aleitamento materno exclusivo, o que ainda afeta na redução da obesidade (WALKER W, 2017).

O cenário ambiental e alimentar da criança é capaz de induzir adaptações das respostas metabólicas, assim como exercer influência sobre o crescimento, a composição corporal e também os riscos de doenças futuras. Estudos apontam que o ambiente nutricional antes do nascimento e durante a primeira infância são de extrema relevância para determinar a saúde futura. Sendo assim, tanto crianças que passaram por condições inadequadas de nutrição durante o período intra uterino, assim como crianças que na vida pós natal não foram amamentadas de forma exclusiva nos seis primeiros meses e/ou tem elevado nível de consumo de nutrientes, possuem risco aumentado para o desenvolvimento de obesidade e diabetes mellitus na infância e na vida adulta (HELLMUTH C, et al., 2016; SYMON B, et al., 2017).

A amamentação é uma prática milenar que influencia diretamente o desenvolvimento infantil, sobretudo nos seis meses iniciais de vida. Segundo a Organização Mundial de Saúde, o aleitamento materno deve acontecer de maneira exclusiva durante os 6 meses de vida e até os 2 anos de idade de forma complementar à alimentação. Contudo, a ausência dessa prática tem repercussões desde a infância até a vida adulta e apresenta grande associação com o surgimento da obesidade. Isso ocorre, devido ao fato que o leite materno é constituído de componentes essenciais que reforçam o sistema imunológico e nutricional, além de favorecer também a maturação intestinal saudável do bebê (ARDID C, 2019).

A presença de imunoglobulinas $(\lg ) \lg A, \lg C, \lg D$, $\lg E$ e $\lg M$, macrófagos, linfócitos, lisozimas e citocinas conferem, gradativamente, um sistema imunológico inato, adaptativo e eficaz que previne e combate infecções patogênicas ao longo da primeira infância e, até mesmo, da vida adulta (ESTÉVEZ-GONZÁLEZ MD, et al., 2016). Outrossim, o perfil hormonal fornecido pelo leite materno através da leptina e adiponectina está associado, respectivamente, à saciedade e ao metabolismo de ácido graxo e sensibilidade à insulina. Em contrapartida, nos bebês alimentados com preparações de fórmulas infantis, além da menor concentração desses hormônios, nota-se um maior índice de grelina que está relacionada à estimulação do apetite (HORN LV, et al., 2018).

Além da inferioridade hormonal, algumas fórmulas infantis possuem altos níveis de proteínas e, por conseguinte, aumentam as concentrações plasmáticas de BCAA (branched-chain amino acids), excedendo a capacidade catabólica normal de uma criança. Dessa forma, a grande oferta de proteínas às crianças pode estimular uma maior secreção de fatores de crescimento semelhante à insulina (IGF-1) e induzir efeitos de sinalização levando ao ganho de peso exagerado. Soma-se a isso o fato de que o consumo de grandes quantidades de proteínas nas fórmulas infantis aparenta inibir a iniciação da $\beta$-oxidação, além de auxiliar no armazenamento de gordura e, portanto, está ligado ao risco aumentado de desenvolvimento de doenças cardiovasculares (HELLMUTH C, et al., 2016).

Como resultado, também foi observado que havia menor risco de obesidade entre as crianças amamentadas por maior período de tempo. $O$ efeito protetor do leite materno tem como uma das justificativas o fato de que seu conteúdo proteico é menor quando comparado a outros tipos de alimentação, como consequência, tem-se menor nível de insulina plasmática, assim como redução do armazenamento de gordura, ou seja, o desenvolvimento precoce de adipócitos é impedido (ESTÉVEZ-GONZÁLEZ MD et al., 2016). Além disso, estudos afirmaram que há uma consonância entre a dieta alimentar oferecida às crianças, sendo eles o aleitamento em si e a introdução alimentar em seus primeiros anos de vida, e o surgimento de sobrepeso até os 3 anos de idade (ARDID C, et al., 2019).

Dentre os componentes do leite materno têm-se também: aminoácidos, vitaminas, minerais e oligossacarídeos. Ademais, a presença de compostos bioativos no leite materno, como os oligossacarídeos, promove a absorção de minerais e o desenvolvimento intestinal, favorecendo a homeostase bacteriana pela multiplicação de microrganismos saudáveis (FICARA M, et al., 2018; WALKER WA, 2017). 
Esses compostos atuam como prebióticos que são substratos alimentares responsáveis por promover o crescimento de espécies benéficas de bactérias, através do fornecimento de carbono. Tais microrganismos auxiliam no processo de digestão, sobretudo o catabolismo de lipídios, auxiliando a taxa de ganho de peso de forma satisfatória e equilibrada. Para além, os oligossacarídeos aumentam a função protetiva da barreira do intestino pela maturação e proliferação das células da cripta e caliciformes, de forma a contribuir, consequentemente, para seletividade da absorção de substâncias e patógenos nesse órgão (FICARA M, et al., 2018; WALKER WA, 2017).

O leite materno é considerado um alimento primordial pelos recém-nascidos até a faixa de 4 a 6 meses de idade. Por outro lado, o leite de vaca não é um alimento recomendado antes do primeiro ano de idade, uma vez que ele pode se pautar com o desenvolvimento de obesidade, alergias, desequilíbrio da pressão arterial, além de afetar o crescimento linear (ZALEWSKI BM, et al., 2015).

Mesmo com o início da ingestão de alimentos complementares, a amamentação pode permanecer de acordo com o pedido do filho, a disponibilidade da mãe ou recomendação do profissional de saúde. Quando há uma nutrição precoce, há chance de ter como desfecho o aumento peso corporal e o comprimento da criança. Logo, existem evidências da relação entre a nutrição no início da vida com a vida adulta, ou seja, podem existir algumas consequências sobre o indivíduo, tais como sobrepeso, obesidade, doenças cardiovasculares, diabetes, alguns tipos de câncer, problemas relacionados à saúde mental, síndromes metabólicas, dentre outras (ZALEWSKI BM, et al., 2015).

Sendo a obesidade uma doença complexa e multifatorial, essa também é fruto de desencadeadores biológicos, sociais e ambientais, assim como é resultado de fatores no início da vida que contribuem de maneira significativa para o seu desenvolvimento, segundo evidências. Dessa forma, o período pré-natal e a fase infantil são períodos cruciais para a determinação do risco de o indivíduo desenvolver tal quadro. Em virtude disso, foi descrito recentemente na literatura o conceito de "Primeiros 1000 dias", cuja explicação se deve ao fato de que, o intervalo desde o período da concepção até os 24 meses de vida é crítico para a indução de desarranjos que tenham como desfecho a obesidade infantil ou tardia. Portanto, intervenções que obtenham a redução de tais riscos como alvo, devem centrar-se neste período de vida (MAMELI C, et al., 2016).

Durante os primeiros 1000 dias, pode-se dividir 3 etapas principais do desenvolvimento alimentar humano, sendo que os fatores de risco podem aparecer em todas. A primeira fase é iniciada com o período pré-natal, já que fatores de risco para a obesidade infantil podem ser encontrados na mãe durante o período gestacional. De acordo com as condições intra-útero, é possível que as mesmas possam programar o feto para ser mais propenso à obesidade ou não. Isso pode ocorrer em razão da exposição aos nutrientes advindos da mãe através da circulação placentária (HELLMUTH C, et al., 2016).

Com isso, medidas preventivas foram elaboradas em busca da redução desse risco durante a gestação. Dentro das medidas preventivas, há um foco inicial que visa a saúde da mãe e do bebê, priorizando a manutenção do peso de ambos durante a gestação, de acordo com a idade gestacional. A adoção de tal medida é para que a chance de obesidade seja diminuída para ambos. Com essa estratégia a manutenção da saúde se torna mais eficaz no pós-parto e assim os fatores de risco serão menos agressivos em outras fases de desenvolvimento (KIRCHBERG FF, et al., 2015; RAMOS-ROMAN MD, et al., 2018).

$\mathrm{Na}$ segunda etapa, os fatores de risco se encontram na fase pós-natal, mais especificamente no tipo de alimentação ofertada ao bebê, ou seja, o leite materno ou as fórmulas. Nesse sentido, é amplamente conhecido o papel protetor do aleitamento materno e o mesmo foi descrito há cerca de 40 anos atrás como uma ação preventiva da obesidade tardia. O leite materno é visto com superioridade nutricional pelos ocidentais quando comparado ao leite em pó, principalmente em termos de prevenção ao ganho de peso. Ao estabelecer uma comparação entre aleitamento materno e o uso de fórmula infantil convencional, conclui-se que a amamentação é capaz de induzir respostas metabólicas e endócrinas diferentes, além de interferir no crescimento, na composição corporal e no risco de doenças na infância e na vida adulta (MAMELI C, et al., 2016; HELLMUTH C, et al., 2016).

Por fim, na terceira e última fase, inclui-se os fatores de risco relacionados à alimentação e dieta precoce. Dentro desse tópico, destaca-se a influência da exposição nutricional das crianças dos 6 meses aos 2 anos 
de idade em relação ao risco de obesidade. Esta etapa é delicada e desafiadora por conta da transição alimentar líquida (leite materno ou fórmula) para a alimentação sólida (MAMELI C, et al., 2016; WALKER WA, 2017).

Portanto, o desmame do lactente deve ocorrer de forma natural, no qual o corpo da criança deve desenvolver uma competência para isso, como: estar adaptado em relação ao desenvolvimento metabólico e imunológico, atento às mudanças epigenéticas, ingerir outros alimentos a fim de ter uma dieta saudável e rica em nutrientes e não mais apresentar interesse nas mamadas. O desmame quando é feito de forma inesperada, pode gerar insegurança para a criança e a mãe, além de poder desencadear uma série de fatores para ambos (MAMELI C, et al., 2016; WALKER WA, 2017).

Em suma, os primeiros 1000 dias, compreendem uma janela de tempo em que os principais fatores de risco para o desenvolvimento da obesidade podem ser identificados. Portanto, o conhecimento dos mecanismos envolvidos e a disseminação de informações sobre o aleitamento materno devem ser alvo de intervenções médicas, uma vez que essas atitudes têm como consequência benefícios na saúde pública, já que tem como objetivo a redução da abrangência da epidemia da obesidade em crianças jovens (MAMELI C, et al., 2016; LOPES AF, et al., 2016).

\section{CONSIDERAÇÕES FINAIS}

O aleitamento materno exclusivo nos primeiros seis meses tem sido um fator protetivo para a obesidade infantil, sendo decorrente da composição do leite materno que auxilia no desenvolvimento do sistema imunológico e da microbiota intestinal do indivíduo. Além disso, há a presença de hormônios relacionados com a saciedade, metabolismo de ácidos graxos e sensibilidade à insulina. Em contrapartida, o uso precoce de fórmulas, com elevada quantidade de proteínas e escassa concentração de hormônios, induz o ganho de peso além do esperado. Dessa forma, é notório que o aleitamento materno é essencial para a prevenção de obesidade e patologias crônicas, proporcionando uma melhor qualidade de vida para a criança.

\section{REFERÊNCIAS}

1. ARDID C, et al. Efectos de las prácticas alimentarias durante la lactancia y de las características maternas en la obesidad infantil. Arch. argent. pediatr, 2019; 26-33, 2019.

2. CORKINS MR, et al. Nutrition in children and adolescents. Medical Clinics, 2016; 100(6):1217-1235.

3. ESTÉVEZ-GONZÁLEZ MD, et al. Breastfeeding during the first 6 months of life, adiposity rebound and overweight/obesity at 8 years of age. International Journal of Obesity, 2016; 40(1): 10-13.

4. FICARA M, et al. Changes of intestinal microbiota in early life. The Journal of Maternal-Fetal \& Neonatal Medicine, 2020; 33(6): 1036-1043.

5. HARRISON M, et al. A qualitative systematic review of maternal infant feeding practices in transitioning from milk feeds to family foods. Maternal \& child nutrition, 2016; 13(2): e12360.

6. HASCHKE F, al. Postnatal high protein intake can contribute to accelerated weight gain of infants and increased obesity risk. Preventive Aspects of Early Nutrition. Karger Publishers, 2016; 85:101-9.

7. HELLMUTH C, et al. Effects of early nutrition on the infant metabolome. Preventive Aspects of Early Nutrition. Karger Publishers, 2016; 85:89-100.

8. KIRCHBERG FF, et al. Dietary protein intake affects amino acid and acylcarnitine metabolism in infants aged 6 months. The Journal of Clinical Endocrinology \& Metabolism, 2015; 100(1): 149-158.

9. LOPES AF, et al. Aleitamento materno, introdução da alimentação complementar e excesso de peso em pré-escolares. ARCHIVOS LATINOAMERICANOS DE NUTRICIÓN, 2016; 66(3): 195-200.

10. MAMELI C, et al. Nutrition in the first 1000 days: the origin of childhood obesity. International Journal of Environmental Research and Public Health, 2016; 13(9): 838.

11. MANNAN $\mathrm{H}$. Early infant feeding of formula or solid foods and risk of childhood overweight or obesity in a socioeconomically disadvantaged region of Australia: a longitudinal cohort analysis. International Journal of Environmental Research and Public Health, 2018; 15(8): 1685.

12. RAMOS-ROMAN MA. Breast milk: A postnatal link between maternal life choices and the prevention of childhood obesity. Clinical therapeutics, 2018; 40(10): 1655-1658.

13. SHAMIR R. The benefits of breast feeding. Protein in Neonatal and Infant Nutrition: Recent Updates. Karger Publishers, 2016; 86: 67-76. 
14. SPECHT IO, et al. Duration of exclusive breastfeeding may be related to eating behaviour and dietary intake in obesity prone normal weight young children. PloS one, 2018; 13(7): e0200388.

15. SYMON B, et al. Does the early introduction of solids promote obesity?. Singapore Medical Journal, 2017; 58(11): 626631.

16. VALDÉS JMB, et al. Exceso de peso y obesidad central y su relación con la duración de la lactancia materna exclusiva. Revista Cubana de Pediatría, 2018; 90(4): e345.

17. HORN LV, et al. Preserving cardiovascular health in young children: beginning healthier by starting earlier. Current atherosclerosis reports, 2018; 20(6): 1-13.

18. WALKER WA. The importance of appropriate initial bacterial colonization of the intestine in newborn, child, and adult health. Pediatric research, 2017; 82(3): 387-395.

19. WEBER $M$, et al. Lower protein content in infant formula reduces BMI and obesity risk at school age: follow-up of a randomized trial. The American Journal of Clinical Nutrition, 2014; 99(5): 041-1051.

20. ZALEWSKI BM, et al. Nutrition of Infants and Young Children (1-3 Years) and its Effect on Later Health: A Systematic Review of Current Recommendations (EarlyNutrition Project), Crit Rev Food Sci Nutr, 2017, 57(3): 489-500. 